growth crosses the middle line it rarely does so directly by way of the vestibule. This is due to the fact that the vestibula mucosa usually escapes the antecedent vulvitisthat is to say, it is not in a precarcinomatous state. Ribbert stated, and we confirm his view, that the surface spread of a carcinoma is at first by successive carcinomatous conversion of the epithelium covering the precarcinomatous area. When this is exhausted further growth continues by division of the cells already formed, but there is no conversion of normal epithelium into carcinoma, the former simply undergoing a pressure destruction owing to the infiltration of the subepithelial tissues by the cells of the growth.

(b) True Multiple Growths.-We have seen four such. In three out of the four they occupied the same side of the vulva, and in none of them do we think that there was any reason to believe that they arose by autoinoculation. In all of them the vulva was lencoplakic, and the double growths were separate by a tract of mucosa that showed the typical changes belonging to that condition. Each separate growth was an entity to itself, starting in its own set of hypertrophic interpapillar processes, and in three of the osses contact between the two tumours was from their position impossible. Moreover, there was no microscopical evidence of any new element having been introduced either from the surface or from the subcutaneous tissue.

Such multiple growths, therefore, should neither be attributed to autoinoculation from the surface nor to metastatic deposit via the subcutaneous tissue. Microscopically it is quite common to find dual, treble, or evon quadruple points of beginning carcinoma. In fact, seeing that every downgrowth begins as a hypertrophic interpapillar epithelial process, the growth may be said always to begin at multiple points.

The rarity of multiple primary growths of the vulva, in spite of the large precarcinomatous area existent; suggests the possibility that the epithelial ingrowth at. the point of the carcinoma may act: as a safety valve to the increased lateral growth temsion that undoubtedly exists throughout the lencoplakic epidermis, and relieves the latter of its tendency to. ingrowth.

Local Recurrences of the Growth.

There are two types of local recurrence. The first is hat in which the original growth has been inefficiently removed, and recurrence takes place in the scar a short time afterwards. We have met with none such. The second is of a different nature, and we have met with examples of this after excision of the valva. It is due to a separate cancer originating almost from a portion of the precarcinomatous area that has escaped removal. It is here to be noted that unless the lines of incision on removal of the vulva for leucoplakic vulvitis are so planned as entirely to be without the diseased area, the leucoplakic condition returns in the scar.

These recrudescences in a portion of leucoplakic epithelium left behind at the original operation are in no way to be confounded with secondary metastatic nodules. Such nodules are subepidermic in origin, whilst the recrudescence is obviously arising from a certain group of hypertrophied interpapillar processes belonging to an area the whole of which is leucoplakic and precarcinomatous (Fig. 8).

Glandular Metastases.

Glandalar involvement occurs late as compared with squamous-celled carcinoms in other sites. The inner set of horizontal inguinal glands are those first affected on one or both sides. When affected they rapidly enlarge, and subsequently often break down. Sections cut from them at this period may fail to demonstrate the nature of the change, so complete is the destruction of the cells that has taken place.

All enlarged glands are not, however, carcinomatous. It has been shown by one of $\mathrm{us}^{8}$ that lymphatic glands prior to their invasion by carcinoma cells are enlarged by a plasma cell proliferation affecting the trabecular elements together with a great hypertrophy of the germinal areas and an increase in number of the lymphocytes derived from them. Glands thus affected may attain a large size, mimicking true carcinomatous enlargement so closely that microscopical investigation is alone competent to decide its nature.
Treatment.

We have tried a large number of remedies for the pruritus in leucoplakic vulvitis, and have found that most of them have failed to give relief. We have had the greatest success with zymocide lotion, resinol ointment, and the $x$ rays. In view, therefore, of our pathological findings, we advise that the affected area should be excised in all cases where these fail, and since we have shown that a permanent change of character has taken place in all the epithelial cells over the affected area, the excision should be a wide one lest the disease recur again in the scar, which has happened to us in two of our cases.

The treatment for kraurosis vulvae is to dissect out the painful parts complained of and enlarge the valval orifice. In carcinoma the inguinal glands on both sides should be removed, whether enlarged or not.

REFERENCES.

! Victor Bonney, Hunterian Lectures. Royal College of Surgeons, and Lancet, 1909. $2 \mathrm{G}$. Thiberge, Etuda clinique sur le kraurosis vulvae Anates de dermatologie et syphilographie, 44 ef .., ix, 1, 17, 1 sos. Lancet, 1908 .

\section{A NEW COLOUR-PERCEPTION SPECTROMETER.}

By F. W. EDRIDGE.GREEN, M.D., F.R.C.S.

THE instrument here described is a spectrometer so arranged as to make it possible to expose to view in the eyepiece the portion of a spectrum between any two desired wave-lengths. It consists (Fig. 1) of the usual

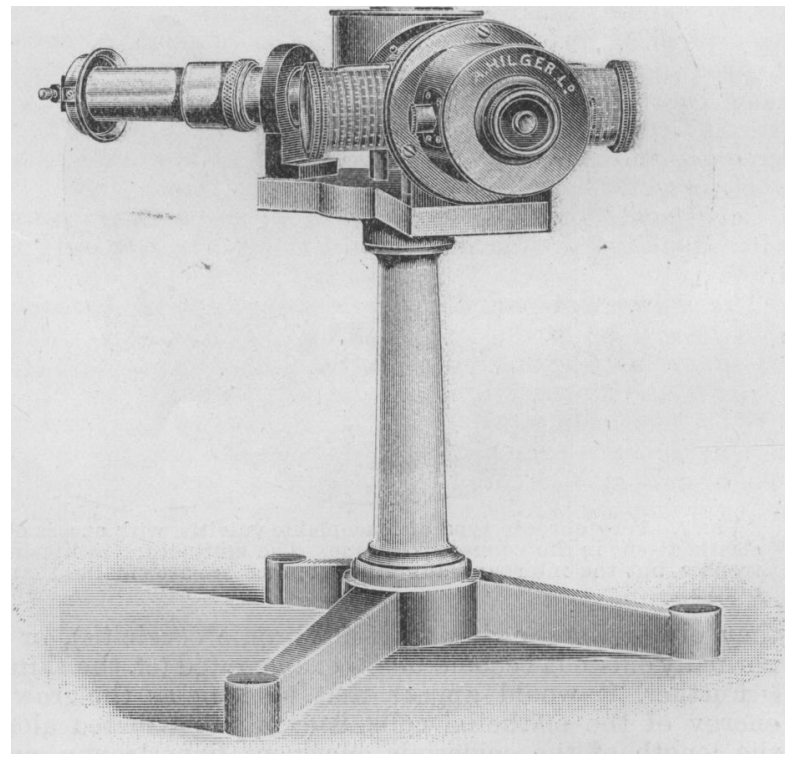

Fig. 1 .

parts of a prism spectroscope-that is, a collimator with adjustable slit, prism, and telescope with eyepiece- of the following dimensions

Focal length of collimator and telescope object glasses $=7 \frac{1}{3}$ in. $(180 \mathrm{~mm}$.). Clear aperture of collimator and telescope

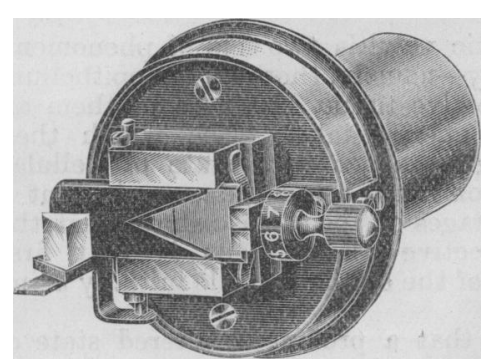

Fig. $2(0.6 \times$ actual size) . object glasses $=\frac{7}{8}$ in (22 mm.). Slit $7 \mathrm{~mm}$. effective length of jaw, with wedge for reducing the effective length of the slit, protective cap, comparison prism. and screw adjustmen for the slit with divided head (see Fig. 2) The head (see Fig. 2). The prism is of flint glass, for 0 . for D. Eyepiece, Ramsden form, focus sing on to the shutter described below.

In the focal plane of the telescope are two adjustable shutters with vertical edges, the shutters being carried by levers which rotate about centres near the object glass of 
the telescope. The shutters can be moved into the field from right and left respectively, each by its owh micro. meter screw, and to each screw is attached a drum, the one being on the right and the other on the left of the telescope. On each of these drums is cut a helical slot in which runs an index, and the drum is engraved (see Fig. 3)

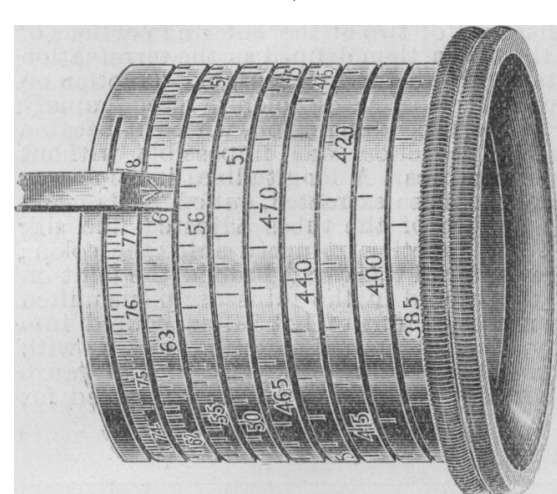

Fig. 3. in such a man. ner that the reading of the index gives the position in the spectrum of the corresponding shutter in wave lengths direct.

Thus, it will be seen that if for instance the reading on the left drum head is 5,320 and that on tho right drumbead is 5,920 , the region of the

spectrum from wave-length 5,320 to wave-length 5,920 is exposed to view in the eyepiece.

An adjustment for the shutters is provided in case of possible zero alterations in course of time. These adjust. ments - which are provided for each shutter independently -are reached by unscrewing the small screw caps on the right and left of the eyepiece end of the telescope. This exposes a screw with a square head, on to which head fits a key which is provided. To adjust the shutter the corresponding drumbead is set to the wave-length of one of the sodium lines. The slit is illuminated by a sodium Hame, and the key is tarned till the edge of the shutter exactly coincides with that line. The key is then removed and the reading checked. The drum will then read correctly throughout the entire spectrum.

Care should, of course, be taken to avoid pressing inward with the key, to which only a turning force should be applied.

The method of estimating colour perception described above will be found in detail in my book on Colour Blindness in the International Scientific Series, and in a paper in the Transactions of the Ophthalmological Society, 1907. It is the method which I originally advocated for testing colour perception, but with the two previous spectrometers devised by me the process was very tedious when quantitative observations were required. The same work can be done with this instrument in less than a twentieth of the time. It is made by A. Hilger, $75 \mathrm{~A}$, Camden Road, N.W.

The instrument should be used as far as possible with a known quality and intensity of light. The observer should first ascertain the exact position of the termination of the red end of the spectrum, the left.hand shatter being moved across until every trace of red just disappears. The position of the pointer on the left-hand drum is noted, and the wave-length recorded. The left drum is then moved so that the shutter is more towards the middle of the spectrum. The right-hand drum is then moved until the pointer indicates the wave-length recorded as the termination of the red end of the spectrum. The abserver then moves the left-hand shutter in and out until he obtains the largest portion of red, which appears absolutely monochromatic to him, no notice being taken of variations in brightness, but only in hue. The position of the pointer on the left-hand drum is recorded. The left-hand shutter is then moved more towares the violet end of the spectrum. The right-hand shutter is then placed at the position previously occupied by the left.hand shatter. In this way the whole of the spectrum is traversed until the termination of the vialet end of the spectrum is finally ascertained with the right-hand shutter. The variation of the size of the patches and the terminations of the spectrum with different intensities of light can be nated. The instrument can also be used for ascertaining the exact position and size of the neutral patch in dichromics, the position of greatest luminosity, and the size and extent of pure colours. When it is used to test colour.blindness, the examinee should first be shown some portion of the interior of the spectrum, and then asked to name the various colours which he sees. In this way he will have no clue to the colours which are being shown him.

The cost of the construction of the instrument was defrayed by a grant from the Royal Society.

\section{TREATMENT OF POST-OPERATIVE SHOCK BY PITUITARY EXTRACT.}

By G. G. WRAY, M.B, Сн.B.Edrs. HOCSE-SURGEON, LOUGHBOROCGH GENERAL HOSPITAL.

The great disadvantage of adrenalin in the treatment of shock is that though it produces great rise in blood-pressure and increase in arterial tone, this effect passes off in an hour or so, and the dose has then to be repeated. Any drug which would produce the same effect without the disadvantages mentioned would be a distinct advance. This I think has been discovered in pituitary extract.

I am indebted to Messrs. Burroughs and Wellcome for the samples of the drug which I used in the three following cases. It is an extract of the posterior lobe of the pituitary body, and is a fluid sterilized in the autoclave, each phial of which contains 1 c.cm. of a 20-per cent. solution.

The contents of one of the phials were injected intra. muscularly into the patient's arm before the patient had fully recovered from the anaesthetic. The effect was almost immediate, and the almost imperceptible pulse soon became large and bounding. This lasted from twelve to sixteen hours, and gradually passed off. Not only did the pulse become larger in expansion but it was also slowed, and whereas it had been irregular it became regular. This effect seems due not only to the action of the drug on the blood vessels, but also on the heart. I gave the injection in conjunction with normal saline per rectum.

CASE I.

A abdomen. She had a swelling on the right side over the appendicular region and extending up above the umbilicus The temperature was $101^{\circ}$, and the pulse 120 . She stated that she had had this pain and temperature for over a week before admission. Abscess of the eppendix wes diagnosed, and immediate laparotomy decided on, as the patient was then somewhat collapsed. On the abdomen being opened an ovarian cyst was found with a twisted pedicle, and which was completely bound down by adhesions at the base; 12 to $15 \mathrm{oz}$. of foul-smelling pus were evacuated. The pedicle was transfixed, the adhesion tied and cut, and the cyst removed. The whole operation took an hour and three-quarters. The patient was put back to bed an hour and three-quarters. The patient was put back to bed in a very collapsed condition : the pulse was small, thready, irregular, and exceedingly difficult to feel. The heart wa fast and irregular, and the apex beat almost imperceptible. five to ten minutes the pulse became gradaslly larger in expanfive to ten minutes the pulse became gradually larger in expanabout a quarter of an hour the pulsation in her temporal about a quarter of an hour the pulsation in her temporal arteries could be seen at some yards' distance. In about half an
hour's time $z x$ of normal saline were given by the rectum, and hour's time $\xi^{x}$ of normal saline were given by the rectum, and the same quantity four-hourly during the night (the operation was finished at 10 p.m.). The next morning the pulse was still but gradually passed off in about fifteen hours. The woman recovered.

CASE II.

A boy, aged 2, was admitted suffering from intussusception of ileo-caecal type, with a history of sixteen hours' duration. An hourafter admission he was operated on and the intussusception reduced. The last portion of the bowel was very difficult to reduce, and was very dark in colour and congested, with a conreduce, and was very dark in colour and congested, with a considerable deposit of lymph. It was returned, and the wound suffering from considerable shock. An injection of 7 minims of pituitary extract was given into the deltoid muscle, and in a few minutes the small, feeble pulse became large, regular, and forceful. Saline was also given by the rectum in small quantities. The effect lasted about twelve hours, and the boy made an uneventful recovery.

CASE III.

A man suffering from Jacksonian epilepsy was trephined, and a considerable area of bone nibbled away over the motor area. A tumour was there found, but proved irremovable. The wound was stitched up, and the patient pat to bed. There was considerable shock. An injection of 1 c.cm. of pituitary extract was given into the deltoid muscle, and the same effect was observed as in the other cases. The man got over his operation, and left the hospital.

In the adults the full dose of 1 c.cm. was given. There were no signs of any ill effects whatsoever. There was 